\title{
PROBABILITY DENSITY FOR A HYPERBOLIC SPDE WITH TIME DEPENDENT COEFFICIENTS*
}

\author{
Marta SAnZ-SOlÉ ${ }^{1}$ AND IVÁn TORREcilla-TARANTino ${ }^{1}$
}

\begin{abstract}
We prove the existence and smoothness of density for the solution of a hyperbolic SPDE with free term coefficients depending on time, under hypoelliptic non degeneracy conditions. The result extends those proved in Cattiaux and Mesnager, PTRF 123 (2002) 453-483 [2] to an infinite dimensional setting.
\end{abstract}

Mathematics Subject Classification. 60H07, 60H15, 60G60.

Received July 10, 2006. Accepted December 26, 2006.

\section{INTRODUCTION}

The initial developments of Malliavin Calculus provided a probabilistic proof of Hörmander's theorem for hypoelliptic operators in square form. As an application, the existence and smoothness of the density for the solution of diffusion processes with coefficients depending only on the spatial variable were obtained (see [5]). There have been several attempts to extend this result to diffusions with coefficients depending on two variables, time and space. The first results in this direction, [3,11], apply to pretty smooth coefficients (see the cases termed in Section 2 as smooth, factorable and regular Hölder). More recently, Cattiaux and Mesnager [2] solved the problem for hypoelliptic coefficients under less restrictive smoothness conditions on the coefficients.

The classical application of Malliavin Calculus mentioned before has been extended in [8] to the two-parameter Itô equation - a wave equation in reduced form. Rules of two-parameter Itô calculus differ from those of the classical one. As a consequence, the analogue of Hörmander's condition is formulated in terms of the covariant derivative instead of the Lie brackets. In view of the results of [2], a natural question is whether one could also extend the results from [8] to coefficients of the equation depending on the two-dimensional time parameter. This article is devoted to study this problem. Combining the techniques of [8] with those of [2], we prove in Theorem 1.1 such an extension.

When studying the inverse of the Malliavin matrix corresponding to homogeneous diffusion processes, one needs estimates of the type

$$
P\left\{\int_{0}^{S} Y_{t}^{2} \mathrm{~d} t \leq a \varepsilon^{\delta}, \int_{0}^{S} \alpha_{t}^{2} \mathrm{~d} t \geq b \varepsilon^{\eta}\right\} \leq \varepsilon^{p},
$$

\footnotetext{
Keywords and phrases. Malliavin calculus. Stochastic partial differential equations. Two-parameter processes.

* Supported by the grant BMF 2003-01345 from the Dirección General de Investigación, Ministerio de Ciencia y Tecnología, Spain.

${ }^{1}$ Facultat de Matemàtiques, Universitat de Barcelona, Gran Via 585, 08007 Barcelona, Spain; marta.sanz@ub.edu; itorrecilla@ub.edu
} 
for small $\varepsilon$ and bounded stopping time $S$. Here $Y_{t}=Y_{0}+M_{t}+V_{t}, t \geq 0$, is a continuous semimartingale with martingale and bounded variation components, $M_{t}$ and $V_{t}$, respectively; it is assumed that the quadratic variation of $M_{t}$ is of the form $\int_{0}^{t} \alpha_{s}^{2} \mathrm{~d} s$ (see for instance $[6,10]$ for different proofs of this result).

When dealing with non-homogeneous diffusions with somehow rough coefficients, such result does not suffice. Actually, the crucial steps of the proofs of Theorem 2.6 and Proposition 3.2 in [2] consist of establishing alternate suitable extensions using a new approach an novel ideas. Setting these ideas in an abstract framework, we prove in Section 2 more sophisticated versions of Stroock-Norris type estimates which are shown to be useful for two-parameter processes.

The paper is organized as follows. In Section 1, we introduce the notation, the different hypothesis we are going to consider along the paper, and we state the main result. Section 2 is devoted to the extension of Stroock-Norris estimates. With these tools, we prove in Section 3 the main result.

\section{Notations, ASsumptions And the main Result}

Consider the stochastic differential equation on $\mathbb{R}^{m}$

$$
X_{z}=x_{0}+\int_{R_{z}}\left[\sum_{l=1}^{d} A_{l}\left(r, X_{r}\right) \mathrm{d} W_{r}^{l}+A_{0}\left(r, X_{r}\right) \mathrm{d} r\right],
$$

where $z=(s, t) \in[0, S] \times[0, T], 0 \leq S, T<\infty, x_{0} \in \mathbb{R}^{m}, R_{z}=[0, s] \times[0, t]$ and $W_{z}=\left(W_{z}^{1}, \ldots, W_{z}^{d}\right)$ is a $d$-dimensional Brownian sheet (see [1]).

Set $\mathcal{E}=\left\{(s, t) \in R_{S, T}: s t \neq 0\right\}$. We assume that the coefficients of (1) satisfy the conditions

(h1) $A_{l}: R_{S, T} \times \mathbb{R}^{m} \longrightarrow \mathbb{R}^{m}, 0 \leq l \leq d$, are $\gamma$-Hölder continuous in $t$, for some $\left.\gamma \in\right] 0,1[$, measurable with respect to $s$, and infinitely differentiable with respect to the spatial variable. Moreover,

$$
K_{\gamma}:=\sup _{y \in \mathbb{R}^{m}} \sup _{0 \leq \theta \leq S} \max _{0 \leq l \leq d}\left\|A_{l}(\theta, \cdot, y)\right\|_{\gamma}<\infty
$$

where the notation $\|\cdot\|_{\gamma}$ refers to the usual Hölder norm.

(h2) for any multi-index $\bar{\alpha}=\left(\alpha_{1}, \ldots, \alpha_{m}\right),|\bar{\alpha}| \geq 0$, and $0 \leq l \leq d$, the partial derivatives $\partial_{\bar{\alpha}} A_{l}$ with respect to $x \in \mathbb{R}^{m}$ exist and

$$
K:=\sup _{0 \leq \theta \leq S} \sup _{0 \leq \tau \leq T} \max _{0 \leq|\bar{\alpha}| \leq N+2} \max _{0 \leq j \leq d}\left\|\partial_{\bar{\alpha}}^{x} A_{j}(\theta, \tau, \cdot)\right\|_{\infty}<\infty
$$

(h3) for a fixed $z=(s, t) \in \mathcal{E}$, the vector fields $A_{l}$ 's, $1 \leq l \leq d$ satisfy the restricted Hörmander's condition stated as follows:

The vector space spanned by the vector fields $A_{1}, \ldots, A_{d}, A_{i}^{\nabla} A_{j}, 1 \leq i, j \leq d, A_{i}^{\nabla}\left(A_{j}^{\nabla} A_{k}\right), 1 \leq$ $i, j, k \leq d, \ldots, A_{i_{1}}^{\nabla}\left(\ldots\left(A_{i_{n-1}}^{\nabla} A_{i_{n}}\right) \ldots\right), 1 \leq i_{1}, \ldots, i_{n} \leq d, \ldots$, at the point $\left(0, t, x_{0}\right)$ has full rank.

Here, the notation $A_{i}^{\nabla} A_{j}$ denotes the covariant derivative of the vector field $A_{j}$ along $A_{i}$.

Assumption (h2) implies that the coefficients of the equation are Lipschitz functions and have linear growth in the spatial variable, uniformly in the time variables. By the usual method of Picard's iterates, one can prove existence and uniqueness of solution for the equation (1) and moreover, the solution has almost surely continuous paths (see Lem. 3.1 in [8]). 
Condition (h3) clearly implies the following. There exist $N:=N\left(x_{0}, t\right) \in \mathbb{N}$, and positive real numbers $c_{N}:=c_{N}\left(x_{0}, t\right), s_{0}:=s_{0}(t), R:=R(t)$ such that for any $v \in S^{m-1}$,

$$
\sum_{k=0}^{N} \sum_{V \in \Sigma_{k}}\langle v, V(\theta, t, y)\rangle^{2} \geq c_{N}
$$

for any $(\theta, y) \in\left[0, s_{0}\right] \times B\left(x_{0}, R\right)$, where $\Sigma_{0}=\left\{A_{l}, 1 \leq l \leq d\right\}, \Sigma_{k}=\left\{A_{l}^{\nabla} V, 1 \leq l \leq d, V \in \Sigma_{k-1}\right\}, k \geq 1$.

We consider different combinations of regularity of the coefficients and non degeneracy conditions of the underlying differential operator, as follows.

(1) Elliptic case: (h1) and (h2) holds. Moreover, the vector space spanned by the vector fields $A_{1}, \ldots, A_{d}$ at the point $\left(0, t, x_{0}\right)$ has full rank.

(2) Smooth case: (h1) to (h3) hold. In addition, for each $0 \leq l \leq d$, the functions $\partial \frac{x}{\alpha} A_{l}$ 's are $C_{b}^{1}$ in $s$, for all multi-index $\bar{\alpha}$,

$$
K_{1}:=\sup _{0 \leq \theta \leq S} \sup _{0 \leq \tau \leq T} \max _{0 \leq|\bar{\alpha}| \leq N} \max _{1 \leq j \leq d}\left\|\partial^{\theta} \partial_{\bar{\alpha}}^{x} A_{j}(\theta, \tau, \cdot)\right\|_{\infty}<\infty
$$

(3) Factorable case: $A_{l}(\theta, \tau, x)=f_{l}(\theta) \bar{A}_{l}(\tau, x), 0 \leq l \leq d$, with $f_{l}$ measurable, $\frac{1}{c^{\prime}} \geq\left|f_{l}\right| \geq c^{\prime}>0$. The functions $\bar{A}_{l}$ 's, $0 \leq l \leq d$, satisfy the analogue of hypothesis (h1)-(h3) for coefficients which do not depend on $\theta$.

(4) Regular Hölder case: (h1) to (h3) hold. In addition, for any multi-index $\bar{\alpha}$, the functions $\partial_{\bar{\alpha}} A_{j}$, $0 \leq j \leq d$, are $\beta(\bar{\alpha})$-Hölder continuous in the argument $s$, for some $\beta(\bar{\alpha}) \in] \frac{1}{2}, 1[$. Moreover,

$$
K_{\beta(\bar{\alpha})}:=\sup _{y \in \mathbb{R}^{m}} \sup _{0 \leq \tau \leq T} \max _{0 \leq|\bar{\alpha}| \leq N+2} \max _{0 \leq l \leq d}\left\|\partial_{\bar{\alpha}}^{x} A_{l}(\cdot, \tau, y)\right\|_{\beta(\bar{\alpha})}<\infty
$$

(5) Irregular Hölder case: The same assumptions as in the preceding case, except that here $\left.\beta(\bar{\alpha}) \in] 0, \frac{1}{2}\right]$.

The main result of this paper is the next theorem, stating the existence and smoothness of density for the probability law of the solution of (1) at any fixed point $z \in \mathcal{E}$.

Theorem 1.1. Let $X=\left\{X_{z}, z \in R_{S, T}\right\}$ be the solution of (1). Each one of the set of assumptions termed before as elliptic, smooth, factorable, regular Hölder and irregular Hölder, imply that the random vector $X_{z}$, for fixed $z \in \mathcal{E}$, has an infinitely differentiable density with respect to the Lebesgue measure.

Remark 1.2. In the formulation of assumptions (h1)-(h3) and of the different scenaries, the roles of the time components $s$ and $t$ might be exchanged.

\section{Stroock-Norris type lemmas For Continuous SEMimartingales DePending ON A PARAMETER}

In this section, we prove two extensions of the Stroock-Norris estimates. The difference between them stands on the order of Hölder continuity of the involved processes. We notice that Lemma 2.1 could provide as a by-product an alternate proof of Norris Lemma [6]. 
Lemma 2.1. Let $\left(Y_{s}(\lambda), s \in[0, S]\right)$ be a real continuous semimartingale depending on a parameter $\lambda \geq 0$, with decomposition

$$
Y_{s}(\lambda)=Y_{0}(\lambda)+M_{s}(\lambda)+V_{s}(\lambda)
$$

where $M_{s}(\lambda), V_{s}(\lambda)$ denote a local martingale and a bounded variation process, respectively, satisfying

$$
\begin{aligned}
M_{s}(\lambda) & =\sum_{j=1}^{m} \int_{0}^{s} \Psi_{\eta}^{j}(\lambda) \mathrm{d} \widetilde{M}_{\eta}^{j}, \quad\left\langle\widetilde{M}^{j}, \widetilde{M}^{k}\right\rangle_{s}=\int_{0}^{s} \Theta_{\eta}^{j, k} \mathrm{~d} \eta, \\
V_{s}(\lambda) & =\int_{0}^{s} \Phi_{\eta}(\lambda) \mathrm{d} \eta .
\end{aligned}
$$

Assume that:

(i) For each $\lambda \geq 0, \Psi_{\eta}^{j}(\lambda), \Phi_{\eta}(\lambda)$ and $\Theta_{\eta}^{j, k}, 1 \leq j, k \leq m$, are adapted continuous processes, indexed by $\eta \in[0, S]$, bounded by some constant $\mathcal{K}$, uniformly in $\eta, \lambda$.

(ii) For each $\eta \in[0, S], 1 \leq j \leq m, Y_{\eta}(\lambda), Y_{0}(\lambda), \Psi_{\eta}^{j}(\lambda), \Phi_{\eta}(\lambda)$ as functions of $\lambda$, are $\beta$-Hölder continuous, with $\frac{1}{2}<\beta<1$, uniformly in $\eta$.

Set $\langle M(\lambda)\rangle_{s}=\int_{0}^{s} \Upsilon_{\eta}(\lambda) \mathrm{d} \eta$, where

$$
\Upsilon_{\eta}(\lambda)=\sum_{j, k=1}^{m} \Psi_{\eta}^{j}(\lambda) \Psi_{\eta}^{k}(\lambda) \Theta_{\eta}^{j, k}
$$

Fix $\nu>\frac{3}{2 \beta-1}$. Then, for any $\rho>3+2 \nu$, positive constants $\alpha_{1}, \alpha_{2}, p \geq 2$, and $\varepsilon$ small enough, there exists a constant $C$ such that

$$
\mathbf{P}\left\{\int_{0}^{s} Y_{u}^{2}(u) \mathrm{d} u \leq \alpha_{1} \varepsilon^{\rho}, \int_{0}^{s} \Upsilon_{u}(u) \mathrm{d} u \geq \alpha_{2} \varepsilon\right\} \leq C \varepsilon^{p} .
$$

Proof. Fix $n \geq 1$ and set $s_{i}=\frac{i s}{n}, i=0, \ldots, n$. For each $i=0, \ldots, n-2$, we define

$$
D_{i}=\left\{\int_{0}^{s} Y_{u}^{2}(u) \mathrm{d} u \leq \alpha_{1} \varepsilon^{\rho}, \int_{s_{i}}^{s_{i+1}} \Upsilon_{u}(u) \mathrm{d} u \geq \frac{\alpha_{2} \varepsilon}{2(n-1)}\right\} .
$$

We also define

$$
D_{n-1}=\left\{\int_{0}^{s} Y_{u}^{2}(u) \mathrm{d} u \leq \alpha_{1} \varepsilon^{\rho}, \int_{\left(1-\frac{1}{n}\right) s}^{s} \Upsilon_{u}(u) \mathrm{d} u \geq \frac{\alpha_{2} \varepsilon}{2}\right\}
$$

Then

$$
\left\{\int_{0}^{s} Y_{u}^{2}(u) \mathrm{d} u \leq \alpha_{1} \varepsilon^{\rho}, \int_{0}^{s} \Upsilon_{u}(u) \mathrm{d} u \geq \alpha_{2} \varepsilon\right\} \subset \bigcup_{i=0}^{n-1} D_{i}
$$

Chebyshev's inequality and the boundedness of $\Psi^{j}, \Theta^{j, k}, 1 \leq j, k \leq m$, yield for all $p^{\prime} \geq 1$,

$$
\mathbf{P}\left\{D_{n-1}\right\} \leq \mathbf{P}\left\{\int_{\left(1-\frac{1}{n}\right) s}^{s} \Upsilon_{u}(u) \mathrm{d} u \geq \frac{\alpha_{2} \varepsilon}{2}\right\} \leq\left(\frac{2 m^{2} \mathcal{K}^{3} s}{\alpha_{2}}\right)^{p^{\prime}} \frac{\varepsilon^{-p^{\prime}}}{n^{p^{\prime}}}
$$

Taking $n=\left[\varepsilon^{-\nu}\right]$ (where [.] denotes the integer part) and $\nu>1$, we have $\frac{\varepsilon^{-p^{\prime}}}{n^{p^{\prime}}} \leq 2^{p^{\prime}} \varepsilon^{(\nu-1) p^{\prime}}$, for any $\varepsilon<\varepsilon_{0}=$ $2^{-\frac{1}{\nu}}$. Therefore,

$$
\mathbf{P}\left\{D_{n-1}\right\} \leq C \varepsilon^{p^{\prime \prime}}
$$

for all $p^{\prime \prime}=(\nu-1) p^{\prime} \geq 2$. 
We now study the terms $\mathbf{P}\left(D_{i}\right)$, for all $i=0, \ldots, n-2$. Setting

$$
F_{i}=\left\{\int_{s_{i}}^{s_{i+2}} Y_{u}^{2}(u) \mathrm{d} u \leq \alpha_{1} \varepsilon^{\rho}, \int_{s_{i}}^{s_{i+1}} \Upsilon_{u}(u) \mathrm{d} u \geq \frac{\alpha_{2} \varepsilon}{2 n}\right\},
$$

we clearly have $D_{i} \subset F_{i}$.

By Itô's formula, for $\left.u \in] s_{i}, s_{i+2}\right]$, it holds that

$$
Y_{u}^{2}(\lambda)=Y_{s_{i}}^{2}(\lambda)+2 \sum_{j=1}^{m} \int_{s_{i}}^{u} Y_{\eta}(\lambda) \Psi_{\eta}^{j}(\lambda) \mathrm{d} \widetilde{M}_{\eta}^{j}+2 \int_{s_{i}}^{u} Y_{\eta}(\lambda) \Phi_{\eta}(\lambda) \mathrm{d} \eta+\int_{s_{i}}^{u} \Upsilon_{\eta}(\lambda) \mathrm{d} \eta
$$

Set $\lambda=u$ in (11). By integrating with respect to the $u$ variable and applying Fubini's theorem and a stochastic Fubini theorem, we obtain

where, for any $s \geq s_{i}$,

$$
\int_{s_{i}}^{s_{i+2}} Y_{u}^{2}(u) \mathrm{d} u \geq \mathcal{C}_{s_{i+2}}+\mathcal{A}_{s_{i+2}}+\mathcal{M}_{s_{i+2}}
$$

$$
\begin{aligned}
\mathcal{C}_{s} & =\int_{s_{i}}^{s}\left(\int_{\eta}^{s} \Upsilon_{\eta}(u) \mathrm{d} u\right) \mathrm{d} \eta \\
\mathcal{A}_{s} & =2 \int_{s_{i}}^{s}\left(\int_{\eta}^{s} Y_{\eta}(u) \Phi_{\eta}(u) \mathrm{d} u\right) \mathrm{d} \eta \\
\mathcal{M}_{s} & =2 \sum_{j=1}^{m} \int_{s_{i}}^{s}\left(\int_{\eta}^{s} Y_{\eta}(u) \Psi_{\eta}^{j}(u) \mathrm{d} u\right) \mathrm{d} \widetilde{M}_{\eta}^{j} .
\end{aligned}
$$

We devote the remaining of the proof to show the inclusion

$$
F_{i} \subset\left\{\sup _{s_{i} \leq u \leq s_{i+2}}\left|\mathcal{M}_{u}\right| \geq \frac{\alpha_{2} s}{16} \varepsilon^{1+2 \nu},\langle\mathcal{M}\rangle_{s_{i+2}}<\frac{\alpha_{2}^{2} s^{2}}{256} \varepsilon^{3+4 \nu}\right\}
$$

for some $\nu>0$. Then, applying the martingale exponential inequality,

$$
\mathbf{P}\left\{\sup _{s_{i} \leq u \leq s_{i+2}}\left|\mathcal{M}_{u}\right| \geq \frac{\alpha_{2} s}{16} \varepsilon^{1+2 \nu},\langle\mathcal{M}\rangle_{s_{i+2}}<\frac{\alpha_{2}^{2} s^{2}}{256} \varepsilon^{3+4 \nu}\right\} \leq 2 \exp \left(-\frac{1}{2} \varepsilon^{-1}\right)
$$

and this will finish the proof.

The above inclusion is obtained by proving a lower bound for $\mathcal{C}_{s_{i+2}}$, and upper bounds for $\left|\mathcal{A}_{s_{i+2}}\right|$ and $\langle\mathcal{M} .\rangle_{s_{i+2}}$.

Lower bound for $\mathcal{C}_{s_{i+2}}$. Clearly, on $F_{i}$, the triangular inequality and the Hölder continuity of $\Upsilon_{\eta}(\cdot)$ (with constant $\mathcal{K}_{\beta}$ ), imply

$$
\begin{aligned}
\mathcal{C}_{s_{i+2}} & \geq \int_{s_{i}}^{s_{i+2}}\left(\int_{\eta}^{s_{i+2}} \Upsilon_{\eta}(\eta) \mathrm{d} u\right) \mathrm{d} \eta-\int_{s_{i}}^{s_{i+2}}\left(\int_{\eta}^{s_{i+2}}\left|\Upsilon_{\eta}(u)-\Upsilon_{\eta}(\eta)\right| \mathrm{d} u\right) \mathrm{d} \eta \\
& \geq \int_{s_{i}}^{s_{i+1}}\left(s_{i+2}-\eta\right) \Upsilon_{\eta}(\eta) \mathrm{d} \eta-\int_{s_{i}}^{s_{i+2}}\left(\int_{\eta}^{s_{i+2}} \mathcal{K}_{\beta}|u-\eta|^{\beta} \mathrm{d} u\right) \mathrm{d} \eta \\
& \geq\left(s_{i+2}-s_{i+1}\right) \int_{s_{i}}^{s_{i+2}} \Upsilon_{\eta}(\eta) \mathrm{d} \eta-\mathcal{K}_{\beta}\left(s_{i+2}-s_{i}\right)^{\beta+2} \\
& \geq \frac{\alpha_{2} s \varepsilon}{2 n^{2}}-\mathcal{K}_{\beta} \frac{(2 s)^{\beta+2}}{n^{\beta+2}} .
\end{aligned}
$$


Taking $n=\left[\varepsilon^{-\nu}\right]$ and $\nu>0$, yields

$$
\frac{\alpha_{2} s \varepsilon}{2 n^{2}}-\mathcal{K}_{\beta} \frac{(2 s)^{\beta+2}}{n^{\beta+2}} \geq \frac{\alpha_{2} s}{2} \varepsilon^{1+2 \nu}-\mathcal{K}_{\beta}\left(4 s \varepsilon^{\nu}\right)^{\beta+2},
$$

for any $\varepsilon<\varepsilon_{0}$. Choose $\nu>0$ such that $\nu \beta>1$, so that $\nu(\beta+2)>1+2 \nu$. Thus, taking $\varepsilon_{1}=\left(\frac{\alpha_{2}}{\mathcal{K}_{\beta} 4^{\beta+3} s^{\beta+1}}\right)^{\frac{1}{\nu \beta-1}}$, we have that for all $\varepsilon<\varepsilon_{1} \wedge \varepsilon_{0}$, on $F_{i}$,

$$
\mathcal{C}_{s_{i+2}} \geq \frac{\alpha_{2} s}{4} \varepsilon^{1+2 \nu}
$$

Upper bound for $\left|\mathcal{A}_{s_{i+2}}\right|$. Jensen's inequality implies

$$
\left|\mathcal{A}_{s_{i+2}}\right| \leq 2 \mathcal{K} \int_{s_{i}}^{s_{i+2}}\left(\int_{\eta}^{s_{i+2}}\left|Y_{\eta}(u)\right| \mathrm{d} u\right) \mathrm{d} \eta
$$

Since $\lambda \rightarrow Y_{u}(\lambda)$ is $\beta$-Hölder continuous (Hölder constant $\mathcal{K}_{\beta}^{\prime}$ ), we obtain

$$
\begin{aligned}
\int_{s_{i}}^{s_{i+2}}\left(\int_{\eta}^{s_{i+2}}\left|Y_{\eta}(u)\right| \mathrm{d} u\right) \mathrm{d} \eta & \leq \int_{s_{i}}^{s_{i+2}}\left(\int_{\eta}^{s_{i+2}}\left|Y_{\eta}(\eta)\right| \mathrm{d} u\right) \mathrm{d} \eta+\int_{s_{i}}^{s_{i+2}}\left(\int_{\eta}^{s_{i+2}}\left|Y_{\eta}(u)-Y_{\eta}(\eta)\right| \mathrm{d} u\right) \mathrm{d} \eta \\
& \leq \int_{s_{i}}^{s_{i+2}}\left(s_{i+2}-\eta\right)\left|Y_{\eta}(\eta)\right| \mathrm{d} \eta+\mathcal{K}_{\beta}^{\prime} \int_{s_{i}}^{s_{i+2}}\left(\int_{\eta}^{s_{i+2}}|u-\eta|^{\beta} \mathrm{d} u\right) \mathrm{d} \eta \\
& \leq \int_{s_{i}}^{s_{i+2}}\left(s_{i+2}-\eta\right)\left|Y_{\eta}(\eta)\right| \mathrm{d} \eta+\mathcal{K}_{\beta}^{\prime}\left(s_{i+2}-s_{i}\right)^{\beta+2} .
\end{aligned}
$$

But,

$$
\begin{aligned}
\int_{s_{i}}^{s_{i+2}}\left(s_{i+2}-\eta\right)\left|Y_{\eta}(\eta)\right| \mathrm{d} \eta & \leq\left\{\int_{s_{i}}^{s_{i+2}}\left(s_{i+2}-\eta\right)^{2} \mathrm{~d} \eta\right\}^{\frac{1}{2}}\left\{\int_{s_{i}}^{s_{i+2}} Y_{\eta}^{2}(\eta) \mathrm{d} \eta\right\}^{\frac{1}{2}} \\
& \leq\left(s_{i+2}-s_{i}\right)^{\frac{3}{2}}\left\{\int_{s_{i}}^{s_{i+2}} Y_{\eta}^{2}(\eta) \mathrm{d} \eta\right\}^{\frac{1}{2}} .
\end{aligned}
$$

Thus, on $F_{i}$, we have

$$
\left|\mathcal{A}_{s_{i+2}}\right| \leq 2 \mathcal{K}\left(\left(\frac{2 s}{n}\right)^{\frac{3}{2}} \alpha_{1}^{\frac{1}{2}} \varepsilon^{\frac{\rho}{2}}+\mathcal{K}_{\beta}^{\prime}\left(\frac{2 s}{n}\right)^{\beta+2}\right) .
$$

As before, taking $n=\left[\varepsilon^{-\nu}\right], \nu>0$, one obtains

$$
\begin{aligned}
\left(\frac{2 s}{n}\right)^{\frac{3}{2}} \alpha_{1}^{\frac{1}{2}} \varepsilon^{\frac{\rho}{2}}+\mathcal{K}_{\beta}^{\prime}\left(\frac{2 s}{n}\right)^{\beta+2} & \leq(4 s)^{\frac{3}{2}} \alpha_{1}^{\frac{1}{2}} \varepsilon^{\frac{3 \nu}{2}+\frac{\rho}{2}}+\mathcal{K}_{\beta}^{\prime}(4 s)^{\beta+2} \varepsilon^{\nu(\beta+2)} \\
& \leq\left((4 s)^{\frac{3}{2}} \alpha_{1}^{\frac{1}{2}}+\mathcal{K}_{\beta}^{\prime}(4 s)^{\beta+2}\right) \varepsilon^{\left(\frac{3 \nu}{2}+\frac{\rho}{2}\right) \wedge(\nu(\beta+2))}
\end{aligned}
$$

for any $\varepsilon<\varepsilon_{0}$. Choose $\nu$ such that $\nu \beta>1$ and $\rho>2+\nu$. Then $m(\rho, \nu, \beta)=\left\{\frac{\rho}{2}+\frac{3 \nu}{2}\right\} \wedge\{\nu(2+\beta)\}>1+2 \nu$. Thus, taking

$$
\varepsilon_{1}^{\prime}=\left(\frac{\alpha_{2}}{32 \mathcal{K}\left(4^{\frac{3}{2}} s^{\frac{1}{2}} \alpha_{1}^{\frac{1}{2}}+\mathcal{K}_{\beta}^{\prime} 4^{\beta+2} s^{\beta+1}\right)}\right)^{\frac{1}{m(\rho, \nu, \beta)-1-2 \nu}},
$$


on $F_{i}$, we obtain for all $\varepsilon<\varepsilon_{1}^{\prime} \wedge \varepsilon_{0}$,

$$
\left|\mathcal{A}_{s_{i+2}}\right| \leq \frac{\alpha_{2} s}{16} \varepsilon^{1+2 \nu}
$$

Upper bound for $\langle\mathcal{M}\rangle_{s_{i+2}}$. Clearly,

$$
\langle\mathcal{M}\rangle_{s_{i+2}}=4 \sum_{j, k=1}^{m} \int_{s_{i}}^{s_{i+2}}\left(\int_{\eta}^{s_{i+2}} Y_{\eta}(u) \Psi_{\eta}^{j}(u) \mathrm{d} u\right)\left(\int_{\eta}^{s_{i+2}} Y_{\eta}(u) \Psi_{\eta}^{k}(u) \mathrm{d} u\right) \Theta_{\eta}^{j, k} \mathrm{~d} \eta
$$

Jensen's inequality implies

$$
\left|\int_{\eta}^{s_{i+2}} Y_{\eta}(u) \Psi_{\eta}^{j}(u) \mathrm{d} u\right| \leq \mathcal{K}\left(s_{i+2}-\eta\right)\left|Y_{\eta}(\eta)\right|+\mathcal{K} \mathcal{K}_{\beta}^{\prime}\left(s_{i+2}-\eta\right)^{\beta+1}
$$

Thus,

$$
\langle\mathcal{M}\rangle_{s_{i+2}} \leq C_{1}\left(s_{i+2}-s_{i}\right)^{2} \int_{s_{i}}^{s_{i+2}} Y_{\eta}^{2}(\eta) \mathrm{d} \eta+C_{2}\left(s_{i+2}-s_{i}\right)^{2 \beta+3}
$$

and on $F_{i}$

$$
\langle\mathcal{M}\rangle_{s_{i+2}} \leq C_{1}\left(\frac{2 s}{n}\right)^{2} \alpha_{1} \varepsilon^{\rho}+C_{2}\left(\frac{2 s}{n}\right)^{2 \beta+3}
$$

Proceeding as before, we may choose $\nu>0$ such that $\nu(2 \beta-1)>3$, then $\rho>3+2 \nu$, and find $\varepsilon_{1}^{\prime \prime}$ such that on $F_{i}$, for all $\varepsilon<\varepsilon_{1}^{\prime \prime}$,

$$
\langle\mathcal{M}\rangle_{s_{i+2}}<\frac{\alpha_{2}^{2} s^{2}}{256} \varepsilon^{3+4 \nu}
$$

The set $F_{i} \cap\left\{\sup _{s_{i} \leq u \leq s_{i+2}}\left|\mathcal{M}_{u}\right|<\frac{\alpha_{2} s}{16} \varepsilon^{1+2 \nu}\right\}$ is empty. In fact, on this set, by (12), (14), (15) and (16), we obtain

$$
\alpha_{1} \varepsilon^{\rho} \geq \int_{s_{i}}^{s_{i+2}} Y_{u}^{2}(u) \mathrm{d} u \geq \mathcal{C}_{s_{i+2}}-\left|\mathcal{A}_{s_{i+2}}\right|-\left|\mathcal{M}_{s_{i+2}}\right| \geq \frac{\alpha_{2} s}{8} \varepsilon^{1+2 \nu}
$$

Hence, taking $\beta>\frac{1}{2}, \nu>\frac{3}{2 \beta-1}, \rho>(3+2 \nu), \varepsilon<\tilde{\varepsilon}_{0}$, with

$$
\tilde{\varepsilon}_{0}:=\min \left\{\left(\frac{\alpha_{2} s}{8 \alpha_{1}}\right)^{\frac{1}{\rho-1-2 \nu}}, \varepsilon_{0}, \varepsilon_{1}, \varepsilon_{1}^{\prime}, \varepsilon_{1}^{\prime \prime}\right\}
$$

(17) cannot be satisfied.

Consequently, for any $\varepsilon<\tilde{\varepsilon}_{0}$,

$$
F_{i}=F_{i} \cap\left\{\sup _{s_{i} \leq u \leq s_{i+2}}\left|\mathcal{M}_{u}\right| \geq \frac{\alpha_{2} s}{16} \varepsilon^{1+2 \nu}\right\} \subset\left\{\sup _{s_{i} \leq u \leq s_{i+2}}\left|\mathcal{M}_{u}\right| \geq \frac{\alpha_{2} s}{16} \varepsilon^{1+2 \nu},\langle\mathcal{M}\rangle_{s_{i+2}}<\frac{\alpha_{2}^{2} s^{2}}{256} \varepsilon^{3+4 \nu}\right\} .
$$

Thus, we obtain (13), and this ends the proof of the lemma. 
The next lemma applies to the more irregular situation where $\left.\beta \in] 0, \frac{1}{2}\right]$.

Lemma 2.2. Consider a continuous semimartingale $\left(Y_{s}(\lambda), 0 \leq s \leq S\right)$, with decomposition given in (7) and satisfying the assumption (i) of Lemma 2.1. Fix $\left.\beta \in] 0, \frac{1}{2}\right]$ and assume that:

(ii') For each $\eta \in[0, S], 1 \leq j \leq m, Y_{0}(\lambda), \Psi_{\eta}^{j}(\lambda), \Phi_{\eta}(\lambda)$, as functions of $\lambda$, are $\beta$-Hölder continuous in $\lambda$, uniformly in $\eta$.

(iii') For all $\beta^{\prime}<\beta$, there exists versions of $\Psi_{\eta}^{j}(\eta)$ and $\Theta_{\eta}^{j, k}, 1 \leq j, k \leq m$, respectively, which are $\beta^{\prime}$-Hölder continuous on $[0, s]$.

Furthermore, for all $p \geq 2$

$$
\mathbb{E}\left(\left\|\Psi^{j}(\cdot)\right\|_{\beta^{\prime}}^{p}+\left\|\Theta^{j, k}(\cdot)\right\|_{\beta^{\prime}}^{p}\right) \leq C_{p}<\infty .
$$

Then, for any $\rho>\left(\frac{11}{2}+\frac{4}{\beta^{\prime}}\right)\left(1+\frac{1}{\beta^{\prime}}\right)$, positive constants $\alpha_{1}, \alpha_{2}, p \geq 2$ and $\varepsilon$ sufficiently small, there exists a constant $C$ such that

$$
\mathbf{P}\left\{\int_{0}^{s} Y_{u}^{2}(u) \mathrm{d} u \leq \alpha_{1} \varepsilon^{\rho}, \int_{0}^{s} \Upsilon_{u}(u) \mathrm{d} u \geq \alpha_{2} \varepsilon\right\} \leq C \varepsilon^{p} .
$$

Proof. We follow the arguments of Proposition 3.2 in [2] in a more abstract presentation.

Fact 1. Fix $\beta^{\prime}<\beta, \varepsilon<s \wedge 1, a>2\left(1+\frac{1}{\beta^{\prime}}\right), p \geq 2$. Condition (ii') implies the existence of a positive constant $c_{p}$ such that

$$
\mathbf{P}\left\{\int_{0}^{s} Y_{u}^{2}(u) \mathrm{d} u \leq \alpha_{1} \varepsilon^{a}, \sup _{0 \leq u \leq s}\left|Y_{u}(u)\right|>\left(1+\sqrt{\alpha_{1}}\right) \varepsilon\right\} \leq c_{p} \varepsilon^{p} .
$$

This can be checked following the proof of Lemma 3.4 on [2] with $\left\langle\xi, \Phi_{u}^{*-1} Y\right\rangle(x):=Y_{u}(u)$.

As a consequence, taking $\rho=r a, \varepsilon^{r}<s \wedge 1$, with $r>0$ and $a$ as before, we reduce the problem to estimate the probability of the set

$$
B=\left\{\sup _{0 \leq u \leq s}\left|Y_{u}(u)\right| \leq\left(1+\sqrt{\alpha_{1}}\right) \varepsilon^{r}, \int_{0}^{s} \Upsilon_{u}(u) \mathrm{d} u \geq \alpha_{2} \varepsilon\right\}
$$

As in the previous Lemma 2.1, we consider the discretization of the interval $[0, s]$ given by $s_{i}=\frac{i s}{n}, i=0, \ldots, n$, with $n=\left[\varepsilon^{-\frac{4}{\beta^{\prime}}}\right]$.

For $i=0, \ldots, n-1$, set

$$
\begin{aligned}
C_{i} & :=\left\{\exists u \in\left[s_{i}, s_{i+1}\right]: \Upsilon_{u}(u)<\alpha_{2} \varepsilon^{\frac{3}{2}}, \int_{0}^{s} \Upsilon_{u}(u) \mathrm{d} u \geq \alpha_{2} \varepsilon\right\}, \\
D_{i} & :=\left\{\sup _{s_{i} \leq u \leq s_{i+1}}\left|Y_{u}(u)\right| \leq\left(1+\sqrt{\alpha_{1}}\right) \varepsilon^{r}, \Upsilon_{u}(u) \geq \alpha_{2} \varepsilon^{\frac{3}{2}}, \forall u \in\left[s_{i}, s_{i+1}\right]\right\} .
\end{aligned}
$$

Clearly, $B \cap D_{i}^{c} \subset C_{i}$.

Fact 2. Condition (iii') implies that for any $p \geq 2$, and $\varepsilon^{\frac{1}{2}}<\frac{1}{2 s}$,

$$
\mathbf{P}\left(\bigcap_{i=0}^{n-1} C_{i}\right) \leq c_{p} \varepsilon^{p} .
$$

For the proof of this fact, we can follow the arguments of Lemma 3.7 in $[2]$ with $\sum_{j=1}^{m}\left\langle\xi, \Phi_{u}^{*-1}\left[X_{j}, Z\right]\right\rangle^{2}(x):=$ $\Upsilon_{u}(u)$. 
Let $D=\bigcup_{i=0}^{n-1} D_{i}$, According to Fact 2, we have $\mathbf{P}\left(B \cap D^{c}\right) \leq c_{p} \varepsilon^{p}$ for any $\varepsilon^{\frac{1}{2}}<\frac{1}{2 s}$.

Therefore, we have only to estimate $\mathbf{P}(B \cap D)$. Moreover, since

$$
\mathbf{P}(B \cap D) \leq \mathbf{P}(D) \leq n \max _{i}\left\{\mathbf{P}\left(D_{i}\right)\right\} \leq \varepsilon^{-\frac{4}{\beta^{\prime}}} \max _{i}\left\{\mathbf{P}\left(D_{i}\right)\right\}
$$

it suffices to show that $\mathbf{P}\left(D_{i}\right) \leq C_{p} \varepsilon^{p}$, for any $p \geq 1$, with some constant $C_{p}$, not depending on $i$.

Recall the definition of $\Upsilon$ given by (8). Owning to assumptions (i) and (ii'),

$$
\sup _{(u, \lambda) \in\left[s_{i}, s_{i+1}\right]^{2}}\left|\Upsilon_{u}(u)-\Upsilon_{u}(\lambda)\right| \leq C\left|s_{i+1}-s_{i}\right|^{\beta}=C\left(\frac{s}{n}\right)^{\beta} \leq C s^{\beta} \varepsilon^{\frac{4 \beta}{\beta^{\prime}}}
$$

for all $\varepsilon<\varepsilon_{0}=2^{-\frac{\beta^{\prime}}{4}}$.

For any $i=0, \ldots, n-1$, set

$$
F_{i}=\left\{\sup _{s_{i} \leq u \leq s_{i+1}}\left|Y_{u}(u)\right| \leq\left(1+\sqrt{\alpha_{1}}\right) \varepsilon^{r}, \inf _{(u, \lambda) \in\left[s_{i}, s_{i+1}\right]^{2}} \Upsilon_{u}(\lambda) \geq \frac{\alpha_{2}}{2} \varepsilon^{\frac{3}{2}}\right\} .
$$

Using (18), we prove that $D_{i} \subset F_{i}$, for all $\varepsilon<\varepsilon_{1} \wedge \varepsilon_{0}$, for some $\varepsilon_{1}>0$.

Indeed, the triangular inequality and the estimate (18) implies that, for any $\lambda \in\left[s_{i}, s_{i+1}\right]$, on $D_{i}$

$$
\Upsilon_{u}(\lambda) \geq \Upsilon_{u}(u)-C s^{\beta} \varepsilon^{\frac{4 \beta}{\beta^{\prime}}} \geq \alpha_{2} \varepsilon^{\frac{3}{2}}-C s^{\beta} \varepsilon^{\frac{4 \beta}{\beta^{\prime}}}
$$

Since $\beta^{\prime}<\beta$, we have $\frac{4 \beta}{\beta^{\prime}}-\frac{3}{2}>0$. Choosing $\varepsilon_{1}=\left(\frac{\alpha_{2}}{2 C s^{\beta}}\right)^{\frac{14}{\beta^{\prime}}-\frac{3}{2}}$, we obtain $\Upsilon_{u}(\lambda) \geq \frac{\alpha_{2}}{2} \varepsilon^{\frac{3}{2}}$, for all $\varepsilon<\varepsilon_{1} \wedge \varepsilon_{0}$ and for any $(u, \lambda) \in\left[s_{i}, s_{i+1}\right]^{2}$.

Thus, we have now reduced the proof to estimate $\mathbf{P}\left\{F_{i}\right\}$.

We shall only consider the case $i=0$. In fact, it will become clear from the proof that the arguments depend only on the length of the interval $\left[s_{i}, s_{i+1}\right]$.

We shall prove the existence of the arg sup associated to $Y .(\lambda)$. This is done following the same arguments as in [2], Proposition 3.2, that is, using Girsanov's theorem. Our setting needs a more general version of this theorem than the one used in [2]. For instance, we can apply Theorem 35 in [9], p. 132. With this, on a new probability space, the semimartingale $Y .(\lambda)$ is transformed into a local martingale and then, by a standard time change, into a Brownian motion, for which the arg sup does exist. We only give a sketch of this procedure, since it is very similar as in [2].

Applying a Girsanov transformation needs to work on the whole probability space $\Omega$, and not only on $F_{0}$. For this reason, we have to modify the process $Y$. $(\lambda)$, as follows. Define

$$
\phi(z)= \begin{cases}z & \text { if }|z| \leq \mathcal{K} \\ \pm 2 \mathcal{K} & \text { if }|z|>3 \mathcal{K}\end{cases}
$$

otherwise, $|\phi|$ is bounded by $2 \mathcal{K}$ and with derivative bounded by 1 ;

$$
\psi_{\varepsilon}(z)= \begin{cases}1 & \text { if }|z| \geq \frac{\alpha_{2}}{2} \varepsilon^{\frac{3}{2}} \\ 0 & \text { if }|z|<\frac{\alpha_{2}}{4} \varepsilon^{\frac{3}{2}}\end{cases}
$$


$\psi_{\varepsilon}$ is even and non-decreasing on $[0, \infty)$. Set

$$
\begin{aligned}
& \bar{\Phi} .(*)=\phi(\Phi .(*)), \\
& \bar{\Psi}^{j}(*)=\phi\left(\Psi^{j}(*)\right) \psi_{\varepsilon}(\Upsilon .(*)), 1 \leq j \leq m, \\
& \bar{\Psi}^{m+1}(*)=\sqrt{\frac{\alpha_{2}}{2} \varepsilon^{\frac{3}{2}}}\left[1-\psi_{\varepsilon}(\Upsilon .(*))\right] .
\end{aligned}
$$

Consider a Brownian motion $\widetilde{M}^{m+1}$, independent of $\left(\widetilde{M}^{1}, \ldots, \widetilde{M}^{m}\right)$. Let

$$
\bar{Y}_{u}(\lambda)=Y_{0}(\lambda)+\sum_{j=1}^{m+1} \int_{0}^{u} \bar{\Psi}_{\eta}^{j}(\lambda) \mathrm{d} \widetilde{M}_{\eta}^{j}+\int_{0}^{u} \bar{\Phi}_{\eta}(\lambda) \mathrm{d} \eta .
$$

This is the analogue of (3.12) in [2].

Observe that if $\omega \in F_{0}$ for $u \leq s_{1}$, then $\bar{Y}_{u}(\lambda, \omega)=Y_{u}(\lambda, \omega)$. Hence, $\bar{Y}$. ( $\left.\lambda\right)$ is a modification of $Y$. $(\lambda)$.

We define the modification of $M_{u}(\lambda)$ by $\bar{M}_{u}(\lambda):=\sum_{j=1}^{m+1} \int_{0}^{u} \bar{\Psi}_{\eta}^{j}(\lambda) \mathrm{d} \widetilde{M}_{\eta}^{j}$, with quadratic variation given by $\langle\bar{M} \cdot(\lambda)\rangle_{u}=\int_{0}^{u} \bar{\Upsilon}_{\eta}(\lambda) \mathrm{d} \eta$, where

$$
\bar{\Upsilon}_{\eta}(\lambda)=\sum_{j, k=1}^{m} \bar{\Psi}_{\eta}^{j}(\lambda) \bar{\Psi}_{\eta}^{k}(\lambda) \Theta_{\eta}^{j, k}+\left(\bar{\Psi}_{\eta}^{m+1}\right)^{2}(\lambda)
$$

One can check that for all $\eta$.

$$
\bar{\Upsilon}_{\eta}(\lambda) \geq \frac{\alpha_{2}}{8} \varepsilon^{\frac{3}{2}}
$$

This is the crucial fact ensuring the existence of a probability measure $\widetilde{\mathbf{P}}$, equivalent to $\mathbf{P}$, such that on each $\mathcal{F}_{s}$,

$$
\frac{\mathrm{d} \widetilde{\mathbf{P}}}{\mathrm{d} \mathbf{P}}=\exp \left\{-\int_{0}^{s} \frac{\bar{\Phi}_{\eta}(\lambda)}{\bar{\Upsilon}_{\eta}(\lambda)} \mathrm{d} \bar{M}_{\eta}(\lambda)-\frac{1}{2} \int_{0}^{s} \frac{\bar{\Phi}_{\eta}^{2}(\lambda)}{\bar{\Upsilon}_{\eta}^{2}(\lambda)} \mathrm{d}\langle\bar{M} \cdot(\lambda)\rangle_{\eta}\right\},
$$

and such that

$$
\overline{\mathbf{M}}_{u}(\lambda)=\bar{M}_{u}(\lambda)+\int_{0}^{u} \bar{\Phi}_{\eta}(\lambda) \mathrm{d} \eta
$$

is a $\widetilde{\mathbf{P}}$ local martingale, (see [9]). Thus, $\widetilde{\mathbf{P}}$-a.s., $\bar{Y}_{u}(\lambda)=Y_{0}(\lambda)+\overline{\mathbf{M}}_{u}(\lambda)$, is a local martingale.

Set

$$
\begin{aligned}
& \mathbf{A}_{u}(\lambda)=\langle\bar{Y} \cdot(\lambda)\rangle_{u}=\int_{0}^{u} \bar{\Upsilon}_{\eta}(\lambda) \mathrm{d} \eta, \\
& \mathbf{T}_{u}(\lambda)=\inf \left\{\eta \geq 0, \mathbf{A}_{\eta}(\lambda) \geq u\right\} .
\end{aligned}
$$

Then, there exists a $\widetilde{\mathbf{P}}$-Brownian motion $B$ such that for all $u, \bar{Y}_{u}(\lambda)=Y_{0}(\lambda)+B_{\mathbf{A}_{u}(\lambda)}$, that is, $\bar{Y}_{\mathbf{T}_{u}(\lambda)}(\lambda)=$ $Y_{0}(\lambda)+B_{u} \widetilde{\mathbf{P}}$-a.s., (see [4], p. 174, for details).

Let $S_{1}=\frac{\alpha_{2} \varepsilon^{\frac{3}{2}}}{8} s_{1} \geq \frac{\alpha_{2}}{8} s \varepsilon^{\frac{3}{2}+\frac{4}{\beta^{\prime}}}$. Using (24), we can prove

$$
u \frac{\alpha_{2}}{8} \varepsilon^{\frac{3}{2}} \leq \mathbf{A}_{u}(\lambda) \leq 2 m^{2} \mathcal{K}^{3} u .
$$


Consequently, $\frac{u}{2 m^{2} \mathcal{K}^{3}} \leq \mathbf{T}_{u}(\lambda) \leq \frac{8 u}{\alpha_{2} \varepsilon^{\frac{3}{2}}}$. Hence,

$$
\frac{\alpha_{2} s \varepsilon^{\frac{3}{2}+\frac{4}{\beta^{\prime}}}}{16 m^{2} \mathcal{K}^{3}} \leq \frac{S_{1}}{2 m^{2} \mathcal{K}^{3}} \leq \mathbf{T}_{S_{1}}(\lambda) \leq \frac{8 S_{1}}{\alpha_{2} \varepsilon^{\frac{3}{2}}}=s_{1}
$$

Set $\Pi_{1}(\lambda):=\mathbf{T}_{S_{1}}(\lambda)$. The supremum of the absolute value of a linear Brownian motion on a deterministic time interval is a.s. attained at a single time. Thus, for all $\lambda \in\left[0, s_{1}\right], \widetilde{\mathbf{P}}$-a.s., there exists an unique (random) time such that

$$
\eta_{1}(\omega, \lambda)=\underset{0 \leq \eta \leq \Pi_{1}(\lambda)}{\arg \sup }\left|\bar{Y}_{\eta}(\lambda)\right|
$$

Since $\mathbf{P}$ and $\widetilde{\mathbf{P}}$ are equivalent and $\Pi_{1}(\lambda) \leq s_{1},(27)$ holds $\mathbf{P}$-a.s.

The final step consists of proving some control on the modified process $\bar{Y} .(\lambda)$, because we only have the existence of the arg sup for this process.

Let $\theta_{1}=\frac{\alpha_{2} s \varepsilon^{\frac{3}{2}+\frac{4}{\beta^{\prime}}}}{16 m^{2} \mathcal{K}^{3}}$. Owing to $(26)$,

$$
\sup _{0 \leq \eta \leq \theta_{1}}\left|\bar{Y}_{\eta}(\lambda)\right| \leq \sup _{0 \leq \eta \leq \Pi_{1}(\lambda)}\left|\bar{Y}_{\eta}(\lambda)\right|
$$

The term $\sup _{0 \leq \eta \leq \theta_{1}}\left|\bar{Y}_{\eta}(\lambda)\right|$ can be estimated in a similar manner as in [6]. We consider the Itô formula for $Y_{\theta_{1}}^{2}(\lambda)$,

$$
\bar{Y}_{\theta_{1}}^{2}(\lambda)=\bar{Y}_{0}^{2}(\lambda)+2 \int_{0}^{\theta_{1}} \bar{Y}_{\eta}(\lambda) \mathrm{d} \bar{M}_{\eta}(\lambda)+2 \int_{0}^{\theta_{1}} \bar{Y}_{\eta}(\lambda) \bar{\Phi}_{\eta}(\lambda) \mathrm{d} \eta+\int_{0}^{\theta_{1}} \bar{\Upsilon}_{\eta}(\lambda) \mathrm{d} \eta
$$

Fix $r^{\prime}>\frac{2}{\beta^{\prime}}+\frac{11}{4}$. We have the following facts concerning $\bar{Y} \cdot(\lambda)$ :

Fact 3. There exists $\varepsilon_{0}^{\prime}>0$ such that for all $\varepsilon<\varepsilon_{0}^{\prime}$

$$
\mathbf{P}\left(\sup _{0 \leq \eta \leq \theta_{1}}\left|\bar{Y}_{\eta}(\lambda)\right| \leq\left(2+\sqrt{\alpha_{1}}\right) \varepsilon^{r^{\prime}}\right) \leq 2 \exp \left(-\frac{1}{2} \varepsilon^{-1}\right)
$$

Fact 4. There exists $\varepsilon_{0}^{\prime \prime}>0$ such that for all $\varepsilon<\varepsilon_{0}^{\prime \prime}$

$$
\mathbf{P}\left(\exists \lambda \in\left[0, s_{1}\right], \sup _{0 \leq \eta \leq \theta_{1}}\left|\bar{Y}_{\eta}(\lambda)\right| \leq\left(1+\sqrt{\alpha_{1}}\right) \varepsilon^{r^{\prime}}\right) \leq c_{p} \varepsilon^{p}
$$

for all $p \geq 2$.

For their proofs, we can follow Lemmas 3.21 and 3.22 of [2], respectively.

Let us return to $Y$. $(\lambda)$. The processes $Y_{u}(\lambda), \mathbf{A}_{u}(\lambda)$ and $\mathbf{T}_{u}(\lambda)$, have jointly continuous sample paths in $(u, \lambda)$, a.s. Thus, for $\omega \in F_{0}$, for all $(u, \lambda) \in\left[0, s_{1}\right]^{2}, \bar{Y}_{u}(\lambda, \omega)=Y_{u}(\lambda, \omega)$. 
Consider the set

$$
G_{0}=F_{0} \cap\left\{\forall \lambda \in\left[0, s_{1}\right], \sup _{0 \leq \eta \leq \Pi_{1}(\lambda)}\left|Y_{\eta}(\lambda)\right|>\left(1+\sqrt{\alpha_{1}}\right) \varepsilon^{r^{\prime}}\right\} .
$$

As in [2], one checks that $G_{0}=\emptyset$. Consequently, with the result stated in Fact 4 , we end the proof of the lemma.

\section{Proof of Theorem 1.1}

Proof. To simplify the notation, we omit the summation sign on repeated indices. Using the classical approach going back to Malliavin -and also by Bismut, Ikeda and Watanabe, Stroock, Bouleau and Hirsch, etc.- (see for instance [7]), the proof of the theorem consists of checking that

(i) $X_{z}^{i} \in \mathbb{D}^{\infty}$, for all $1 \leq i \leq m$;

(ii) $\operatorname{det} C_{z}^{-1} \in L^{p}$, for all $p \geq 2$, where $C_{z}$ is the $m \times m$ matrix whose entries are

$$
C_{z}^{i j}=\int_{R_{z}}\left(D_{r}^{l} X_{z}^{i}\right)\left(D_{r}^{l} X_{z}^{j}\right) \mathrm{d} r
$$

Proving (i) is straightforward. Indeed, due to the condition (h2), one can proceed as in [8], Proposition 3.3.

To prove (ii), it suffices to show that for any $p \geq 2$ there exists $\varepsilon_{0}(p)$ such that for every $\varepsilon \leq \varepsilon_{0}(p)$

$$
\sup _{|v|=1} \mathbf{P}\left\{v^{T} C_{z} v \leq \varepsilon\right\} \leq \varepsilon^{p}
$$

(see e.g. [7]).

We next give some preparations for the proof of (30), valid in each one of the set of assumptions of Theorem 1.1. Following similar arguments as in [8], pp. 585-586, we write

$$
v^{T} C v=\sum_{l=1}^{d} \int_{R_{z}}\left\langle v, \xi(r, z) A_{l}\left(r, X_{r}\right)\right\rangle^{2} \mathrm{~d} r
$$

where $v \in \mathbb{R}^{m}$, and for $r \in R_{S, T}, \xi_{j}^{i}(r, z), r \preceq z, 1 \leq i, j \leq m$, is the solution to

$$
\xi_{j}^{i}(r, z)=\delta_{j}^{i}+\int_{[r, z]} \partial_{k}^{x} A_{l}^{i}\left(u, X_{u}\right) \xi_{j}^{k}(r, u) \mathrm{d} W_{u}^{l}+\int_{[r, z]} \partial_{k}^{x} A_{0}^{i}\left(u, X_{u}\right) \xi_{j}^{k}(r, u) \mathrm{d} u .
$$

Then, for any $v \in S^{m-1}, 0<\varepsilon<1,0<\mu<1$, we have

$$
\mathbf{P}\left\{v^{T} C_{z} v \leq \varepsilon\right\} \leq \mathbf{P}\left(E_{0}\right)+\mathbf{P}(B),
$$

where

$$
\begin{aligned}
E_{0} & =\left\{\sum_{l=1}^{d} \int_{0}^{s}\left\langle v, A_{l}\left(\eta, t, X_{\eta, t}\right)\right\rangle^{2} \mathrm{~d} \eta \leq 4 \varepsilon^{\mu}\right\} \\
B & =\left\{\sum_{l=1}^{d} \int_{0}^{s} \int_{t-\varepsilon^{1-\mu}}^{t}\left\langle v, A_{l}\left(\eta, t, X_{\eta, t}\right)-\xi(\eta, \tau, s, t) A_{l}\left(\eta, \tau, X_{\eta, \tau}\right)\right\rangle^{2} \mathrm{~d} \eta \mathrm{d} \tau>\varepsilon\right\} .
\end{aligned}
$$


Combining results of [8] and the assumption (h1), we can obtain an upper bound like in (30) for $P(B)$, as follows. Applying Chebyshev and Cauchy-Schwarz inequalities yields

$$
\mathbf{P}\{B\} \leq C \varepsilon^{-\mu q} \sup _{(\eta, \tau) \in[0, s] \times\left[t-\varepsilon^{1-\mu}, t\right]} \max _{1 \leq l \leq d} \mathbb{E}\left\|A_{l}\left(\eta, t, X_{\eta, t}\right)-\xi(\eta, \tau, s, t) A_{l}\left(\eta, \tau, X_{\eta, \tau}\right)\right\|^{2 q} .
$$

Clearly,

$$
\begin{aligned}
\mathbb{E}\left\|A_{l}\left(\eta, t, X_{\eta, t}\right)-\xi(\eta, \tau, s, t) A_{l}\left(\eta, \tau, X_{\eta, \tau}\right)\right\|^{2 q} \leq & C \mathbb{E}\left\|A_{l}\left(\eta, t, X_{\eta, t}\right)-A_{l}\left(\eta, \tau, X_{\eta, t}\right)\right\|^{2 q} \\
& +C \mathbb{E}\left\|A_{l}\left(\eta, \tau, X_{\eta, t}\right)-A_{l}\left(\eta, \tau, X_{\eta, \tau}\right)\right\|^{2 q} \\
& +C \mathbb{E}\left\|A_{l}\left(\eta, \tau, X_{\eta, \tau}\right)-\xi(\eta, \tau, s, t) A_{l}\left(\eta, \tau, X_{\eta, \tau}\right)\right\|^{2 q} .
\end{aligned}
$$

From (h1), we have

$$
\mathbb{E}\left\|A_{l}\left(\eta, t, X_{\eta, t}\right)-A_{l}\left(\eta, \tau, X_{\eta, t}\right)\right\|^{2 q} \leq K_{\gamma}^{2 q}|t-\tau|^{2 q \gamma}
$$

By the mean value theorem in the spatial component and Lemma 3.1 in [8] applied to (1),

$$
\mathbb{E}\left\|A_{l}\left(\eta, \tau, X_{\eta, t}\right)-A_{l}\left(\eta, \tau, X_{\eta, \tau}\right)\right\|^{2 q} \leq C|t-\tau|^{q} .
$$

Finally, the Cauchy-Schwarz inequality and Lemma 3.1 in [8] applied to (31) yield

$$
\begin{aligned}
& \mathbb{E}\left\|A_{l}\left(\eta, \tau, X_{\eta, \tau}\right)-\xi(\eta, \tau, s, t) A_{l}\left(\eta, \tau, X_{\eta, \tau}\right)\right\|^{2 q} \\
& =\mathbb{E}\left\|\left(I_{m}-\xi(\eta, \tau, s, t)\right) A_{l}\left(\eta, \tau, X_{\eta, \tau}\right)\right\|^{2 q} \\
& \leq C|(s-\eta)(t-\tau)|^{q} .
\end{aligned}
$$

Consequently,

$$
\mathbf{P}\{B\} \leq C \varepsilon^{\{(1-2 \mu) \wedge(2 \gamma-(2 \gamma+1) \mu)\} q} .
$$

If $\mu<\frac{1}{2} \wedge \frac{2 \gamma}{2 \gamma+1}$ then $(1-2 \mu) \wedge(2 \gamma-(2 \gamma+1) \mu)>0$. Thus, in this case,

$$
\mathbf{P}\{B\} \leq C\left(K, K_{\gamma}, S, T, d, p\right) \varepsilon^{p},
$$

for all $p=\{(1-2 \mu) \wedge(2 \gamma-(2 \gamma+1) \mu)\} q \geq 2$.

We devote the remaining of the section to estimate the term $\mathbf{P}\left(E_{0}\right)$.

For any $k=0,1, \ldots, N, v \in S^{m-1}$, define

$$
E_{k}:=\left\{\sum_{V \in \Sigma_{k}} \int_{0}^{s}\left\langle v, V\left(\eta, t, X_{\eta, t}\right)\right\rangle^{2} \mathrm{~d} \eta \leq 4 \varepsilon^{m(k)}\right\}
$$

and $E=\underset{k=0}{N} E_{k}$, where the $m(k)$ are positive constants to be fixed later.

As usually, for $N \geq 1$ we consider the decomposition $E_{0} \subset\left(E_{0} \cap E_{1}^{c}\right) \cup\left(E_{1} \cap E_{2}^{c}\right) \cup \cdots \cup\left(E_{N-1} \cap E_{N}^{c}\right) \cup E$, yielding

$$
\mathbf{P}\left(E_{0}\right) \leq \mathbf{P}(E)+\sum_{k=0}^{N-1} \mathbf{P}\left(E_{k} \cap E_{k+1}^{c}\right) .
$$

We are going to estimate each term of this sum under the different set of assumptions of the theorem.

Assume (h3); we shall prove that $\mathbf{P}(E) \leq C \varepsilon^{p}$, for any $\varepsilon>0$ small enough, where $C$ depends on $t, p, m, K$ and $R$. 
Consider the stopping time $\mathcal{S}$ with respect to the family of $\sigma$-algebras $\left\{\mathcal{F}_{\eta, t}, \eta \geq 0\right\}$ (associated to the Brownian sheet) defined as

$$
\mathcal{S}=\inf \left\{\eta \geq 0: \sup _{\theta \leq \eta ; \tau \leq t}\left|X_{\theta, \tau}-x_{0}\right| \geq R\right\} \wedge s \wedge s_{0} .
$$

We write $\mathbf{P}(E) \leq \mathbf{P}\left(E \cap\left\{\mathcal{S} \geq \varepsilon^{\varsigma}\right\}\right)+\mathbf{P}\left(\mathcal{S}<\varepsilon^{\varsigma}\right)$, with $0<\varsigma<M=\min _{k=0, \ldots, N} m(k)$.

For $\varepsilon$ small enough, the set $E \cap\left\{\mathcal{S} \geq \varepsilon^{\varsigma}\right\}$ is empty. In fact, assume $\mathcal{S} \geq \varepsilon^{\varsigma}$; from (4) we have

$$
\sum_{k=0}^{N} \sum_{V \in \Sigma_{k}} \int_{0}^{s}\left\langle v, V\left(\eta, t, X_{\eta, t}\right)\right\rangle^{2} \mathrm{~d} \eta \geq c_{N} \varepsilon^{\varsigma}
$$

On the set $E$,

$$
\sum_{k=0}^{N} \sum_{V \in \Sigma_{k}} \int_{0}^{s}\left\langle v, V\left(\eta, t, X_{\eta, t}\right)\right\rangle^{2} \mathrm{~d} \eta \leq 4(N+1) \varepsilon^{M}
$$

Thus, for all $\varepsilon<\varepsilon_{0}=\left(\frac{c_{N}}{4(N+1)}\right)^{\frac{1}{M-\varsigma}}, E \cap\left\{\mathcal{S} \geq \varepsilon^{\varsigma}\right\}=\emptyset$.

Applying Chebychev, Burkholder-Davis-Gundy and Hölder inequalities, Gronwall's lemma and Lemma 3.1 in [8] to (1), yields $\mathbf{P}\left(\mathcal{S}<\varepsilon^{\varsigma}\right) \leq C \varepsilon^{\frac{p^{\prime} \varsigma}{2}}$, for all $p^{\prime} \geq 2$. Thus, taking $p=\frac{p^{\prime} \varsigma}{2} \geq 2$, for all $\varepsilon<\varepsilon_{0}$

$$
\mathbf{P}(E) \leq \mathbf{P}\left(\mathcal{S}<\varepsilon^{\varsigma}\right) \leq C \varepsilon^{p},
$$

where $C$ depends on $p, t, m, K$ and $R$.

Assume $N=0$. Then $E=E_{0}$; therefore, for the elliptic case the proof is complete.

\section{The smooth case}

In addition to the results proved so far, we have to study $\mathbf{P}\left(E_{k} \cap E_{k+1}^{c}\right), k=0, \ldots, N-1$. Clearly, $E_{k} \cap E_{k+1}^{c} \subset \cup_{V \in \Sigma_{k}} B$, where

$$
B=\left\{\int_{0}^{s}\left\langle v, V\left(\eta, t, X_{\eta, t}\right)\right\rangle^{2} \mathrm{~d} \eta \leq 4 \varepsilon^{m(k)}, \sum_{l=1}^{d} \int_{0}^{s}\left\langle v, A_{l}^{\nabla} V\left(\eta, t, X_{\eta, t}\right)\right\rangle^{2} \mathrm{~d} \eta>\frac{4 \varepsilon^{m(k+1)}}{\Gamma}\right\},
$$

and $\Gamma=\max _{k=0, \ldots, N-1} \operatorname{card}\left(\Sigma_{k}\right)$.

Moreover, $B \subset B^{1} \cup B^{2}$, with

$$
\begin{aligned}
& B^{1}:=\left\{\int_{0}^{s}\left\langle v, V\left(\eta, t, X_{\eta, t}\right)\right\rangle^{2} \mathrm{~d} \eta \leq 4 \varepsilon^{m(k)}, \sum_{l=1}^{d} \int_{0}^{s} \int_{0}^{t}\left\langle v, A_{l}\left(\eta, \tau, X_{\eta, \tau}\right)^{\nabla} V\left(\eta, t, X_{\eta, t}\right)\right\rangle^{2} \mathrm{~d} \eta \mathrm{d} \tau \geq \frac{\varepsilon^{\vartheta m(k+1)}}{\Gamma}\right\}, \\
& B^{2}:=\left\{\sum_{l=1}^{d} \int_{0}^{s} \int_{0}^{t}\left\langle v, A_{l}\left(\eta, \tau, X_{\eta, \tau}\right) \nabla\left(\eta, t, X_{\eta, t}\right)\right\rangle^{2} \mathrm{~d} \eta \mathrm{d} \tau<\frac{\varepsilon^{\vartheta m(k+1)}}{\Gamma},\right. \\
&\left.\sum_{l=1}^{d} \int_{0}^{s}\left\langle v, A_{l}^{\nabla} V\left(\eta, t, X_{\eta, t}\right)\right\rangle^{2} \mathrm{~d} \eta>\frac{4 \varepsilon^{m(k+1)}}{\Gamma}\right\},
\end{aligned}
$$

where $\vartheta$ is an arbitrary positive real constant. 
Let us estimate $\mathbf{P}\left(B^{2}\right)$. Firstly, one easily checks that $B^{2} \subset B^{2^{\prime}}$ with

$$
B^{2^{\prime}}=\left\{\sum_{l=1}^{d} \int_{0}^{s} \int_{t-\varepsilon^{(\vartheta-1) m(k+1)}}^{t}\left\langle v,\left\{A_{l}\left(\eta, t, X_{\eta, t}\right)-A_{l}\left(\eta, \tau, X_{\eta, \tau}\right)\right\}^{\nabla} V\left(\eta, t, X_{\eta, t}\right)\right\rangle^{2} \mathrm{~d} \eta \mathrm{d} \tau>\frac{\varepsilon^{\vartheta m(k+1)}}{\Gamma}\right\} .
$$

Applying Chebyshev's and the Cauchy-Schwarz inequalities, yields

$$
\mathbf{P}\left(B^{2^{\prime}}\right) \leq C \varepsilon^{-q m(k+1)} \sup _{\substack{\eta \in[0, s] \\ \tau \in\left[t-\varepsilon^{(\vartheta-1) m(k+1)}, t\right]^{1 \leq j \leq m}}} \max _{\substack{1 \leq l \leq d \\ 1 \leq j \leq}}\left\{\mathbb{E}\left\|A_{l}\left(\eta, t, X_{\eta, t}\right)-A_{l}\left(\eta, \tau, X_{\eta, \tau}\right)\right\|^{4 q} \mathbb{E}\left\|\partial_{j}^{x} V\left(\eta, t, X_{\eta, t}\right)\right\|^{4 q}\right\}^{\frac{1}{2}}
$$

We have $\mathbb{E}\left\|\partial_{j}^{x} V\left(\eta, t, X_{\eta, t}\right)\right\|^{4 q} \leq K^{4 q}$. Moreover, owing to (32) and (33),

$$
\mathbb{E}\left\|A_{l}\left(\eta, t, X_{\eta, t}\right)-A_{l}\left(\eta, \tau, X_{\eta, \tau}\right)\right\|^{4 q} \leq 2^{4 q-1}\left(K_{\gamma}^{4 q}|t-\tau|^{4 q \gamma}+C(K, S, T, q)|t-\tau|^{2 q}\right) .
$$

Thus, $\mathbf{P}\left(B^{2}\right) \leq C \varepsilon^{\{(2(\vartheta-1) \gamma-1) \wedge(\vartheta-2)\} q m(k+1)}$. Taking $\vartheta>\left(\frac{1}{2 \gamma}+1\right) \vee 2$, we obtain $\{(2(\vartheta-1) \gamma-1) \wedge(\vartheta-2)\}>$ 0. Hence, $\mathbf{P}\left(B^{2}\right) \leq C \varepsilon^{p}$, for all $p=\{(2(\vartheta-1) \gamma-1) \wedge(\vartheta-2)\} q m(k+1) \geq 2$.

It remains to study the term $\mathbf{P}\left(B^{1}\right)$. For fixed $t$, consider the one-parameter semimartingale $\left(X_{s, t}, s \in[0, S]\right)$ with respect to the filtration $\left\{\mathcal{F}_{\eta, t}, \eta \geq 0\right\}$.

An application of the Itô formula yields

$$
\begin{aligned}
\left\langle v, V\left(s, t, X_{s, t}\right)\right\rangle= & \left\langle v, V\left(0, t, x_{0}\right)\right\rangle+\int_{0}^{s}\left\langle v, \partial^{\eta} V\left(\eta, t, X_{\eta, t}\right)\right\rangle \mathrm{d} \eta \\
& +\sum_{l=1}^{d} \int_{0}^{s} \int_{0}^{t}\left\langle v, A_{l}\left(\eta, \tau, X_{\eta, \tau}\right)^{\nabla} V\left(\eta, t, X_{\eta, t}\right)\right\rangle \mathrm{d} W_{\eta, \tau}^{l} \\
& +\int_{0}^{s} \int_{0}^{t}\left\langle v, A_{0}\left(\eta, \tau, X_{\eta, \tau}\right)^{\nabla} V\left(\eta, t, X_{\eta, t}\right)\right\rangle \mathrm{d} \eta \mathrm{d} \tau \\
& +\frac{1}{2} \int_{0}^{s} \int_{0}^{t}\left\langle v, \partial_{j}^{x} \partial_{k}^{x} V\left(\eta, t, X_{\eta, t}\right) A_{l}^{j}\left(\eta, \tau, X_{\eta, \tau}\right) A_{l}^{k}\left(\eta, \tau, X_{\eta, \tau}\right)\right\rangle \mathrm{d} \eta \mathrm{d} \tau .
\end{aligned}
$$

We can now use the arguments of [8]. More precisely, we apply Lemma 4.2 in [8] to the continuous semimartingale $Y_{s}:=\left\langle v, V\left(s, t, X_{s, t}\right)\right\rangle$, which decomposition follows from (35). This finishes the proof in the regular case.

The estimate of $P\left(E_{0}\right)$ in the factorable case can be obtained using the same method as for the regular case. We skip the details of the proof to avoid repetitions.

\section{The regular Hölder case}

Under this set of assumptions, the expression (35) does not make sense, because the vector fields $V(\eta, t, x)$ are not differentiable with respect to the variable $\eta$. Instead, we consider the process $\left(\left\langle v, V\left(\lambda, t, X_{\eta, t}\right), \eta \in[0, s]\right)\right.$, where $V \in \Sigma_{k}, k=0, \ldots, N-1$, and $\lambda \geq 0, t \in[0, T]$, are fixed, and apply the Itô formula. We obtain

$$
\begin{aligned}
\left\langle v, V\left(\lambda, t, X_{s, t}\right)\right\rangle= & \left\langle v, V\left(\lambda, t, x_{0}\right)\right\rangle+\sum_{l=1}^{d} \int_{0}^{s} \int_{0}^{t}\left\langle v, A_{l}\left(\eta, \tau, X_{\eta, \tau}\right)^{\nabla} V\left(\lambda, t, X_{\eta, t}\right)\right\rangle \mathrm{d} W_{\eta, \tau}^{l} \\
& +\int_{0}^{s} \int_{0}^{t}\left\langle v, A_{0}\left(\eta, \tau, X_{\eta, \tau}\right)^{\nabla} V\left(\lambda, t, X_{\eta, t}\right)\right\rangle \mathrm{d} \eta \mathrm{d} \tau \\
& +\frac{1}{2} \int_{0}^{s} \int_{0}^{t}\left\langle v, \partial_{j}^{x} \partial_{k}^{x} V\left(\lambda, t, X_{\eta, t}\right) A_{l}^{j}\left(\eta, \tau, X_{\eta, \tau}\right) A_{l}^{k}\left(\eta, \tau, X_{\eta, \tau}\right)\right\rangle \mathrm{d} \eta \mathrm{d} \tau .
\end{aligned}
$$


Set

$$
\begin{aligned}
Y_{s}(\lambda)= & \left\langle v, V\left(\lambda, t, X_{s, t}\right)\right\rangle, \quad Y_{0}(\lambda)=\left\langle v, V\left(\lambda, t, x_{0}\right)\right\rangle, \\
\Psi_{\eta}^{j}(\lambda)= & \left\langle v, \partial_{j}^{x} V\left(\lambda, t, X_{\eta, t}\right)\right\rangle, \quad \widetilde{M}_{s}^{j}=\int_{0}^{s} \int_{0}^{t} A_{l}^{j}\left(\eta, \tau, X_{\eta, \tau}\right) \mathrm{d} W_{\eta, \tau}^{l}, \\
\Theta_{\eta}^{j, k}= & \int_{0}^{t} \sum_{l=1}^{d} A_{l}^{j}\left(\eta, \tau, X_{\eta, \tau}\right) A_{l}^{k}\left(\eta, \tau, X_{\eta, \tau}\right) \mathrm{d} \tau, \\
\Phi_{\eta}(\lambda)= & \int_{0}^{t}\left\langle v, A_{0}\left(\eta, \tau, X_{\eta, \tau}\right)^{\nabla} V\left(\lambda, t, X_{\eta, t}\right)\right\rangle \mathrm{d} \tau \\
& +\frac{1}{2} \int_{0}^{t}\left\langle v, \partial_{j}^{x} \partial_{k}^{x} V\left(\lambda, t, X_{\eta, t}\right) A_{l}^{j}\left(\eta, \tau, X_{\eta, \tau}\right) A_{l}^{k}\left(\eta, \tau, X_{\eta, \tau}\right)\right\rangle \mathrm{d} \tau,
\end{aligned}
$$

and $\alpha_{1}=4, \alpha_{2}=\frac{1}{\Gamma}$.

These processes satisfy the assumptions of Lemma 2.1. Fix $\nu>\frac{3}{2 \beta-1}$, then $3+2 \nu<\rho$. Set $m(0)=\mu$ and $m(k)=\frac{\mu}{(\theta \rho)^{k}}, k=0, \ldots, N$. With Lemma 2.1, we obtain the desired estimate for $P\left(B^{1}\right)$ and we finish the proof.

\section{The irregular Hölder case}

We apply Lemma 2.2 to the same processes as we did in the regular Hölder case. Notice that the assumptions of this lemma are satisfied. Fix $\rho>\left(\frac{11}{2}+\frac{4}{\beta^{\prime}}\right)\left(1+\frac{1}{\beta^{\prime}}\right)$. Set $m(0)=\mu$ and $m(k)=\frac{\mu}{(\vartheta \rho)^{k}}, k=0, \ldots, N$. Then, for $\varepsilon$ small enough we obtain the suitable estimate for $P\left(B^{1}\right)$, and therefore the proof is complete.

Acknowledgements. The authors would like to thank P. Cattiaux and L. Mesnager for crucial discussions on some aspects of the proof of Lemma 2.1. A part of this work has been done while the first author was visiting the Centro di Recerca Matematica Ennio De Giorgi at Pisa. She would like to express her gratitude for the warm hospitality and the financial support provided by the Centre.

\section{REFERENCES}

[1] R. Cairoli and J.B. Walsh, Stochastic integrals in the plane. Acta Mathematica 134 (1975) 111-183.

[2] P. Cattiaux and L. Mesnager, Hypoelliptic non-homogeneous diffusions. PTRF 123 (2002) 453-483.

[3] M. Chen and X. Zhou, Applications of Malliavin calculus to stochastic differential equations with time-dependent coefficients. Acta Appli. Math. Sinica 7 (1991) 193-216.

[4] I. Karatzas and S.E. Shreve, Brownian Motion and Stochastic Calculus. Springer-Verlag (1988).

[5] P. Malliavin, Stochastic calculus of variations and hypoelliptic operators, in Proc. Inter. Symp. on Stoch. Diff. Equations, Kyoto 1976, Tokyo and Wiley, New York (1978) 195-263.

[6] J.R. Norris, Simplified Malliavin calculus, in Séminaire de Probabilités XX. LNM 1204 (1986) 101-130.

[7] D. Nualart, The Malliavin Calculus and Related Topics. Probability and its Applications. Springer-Verlag, 2nd Edition (2006).

[8] D. Nualart and M. Sanz, Malliavin calculus for two-parameter Wiener functionals. Z. für Wahrscheinlichkeitstheorie verw. Gebiete 70 (1985) 573-590.

[9] P.E. Protter, Stochastic Integration and Differential Equations. Applications of Mathematics. Stochastic Modelling and Applied Probability. Springer, 2nd Edition 21 (2004).

[10] D.W. Stroock, Some applications of stochastic calculus to partial differential equations, in École d'Été de Probabilités de Saint Flour. LNM 976 (1983) 267-382.

[11] S. Taniguchi, Applications of Malliavin's calculus to time-dependent systems of heat equations. Osaka J. Math. 22 (1985) 307-320. 